\title{
Les réarrangements du gène NPM en 5q35: un élément nouveau dans la compréhension et le démembrement des lymphomes anaplasiques à grandes cellules, CD30+
}

\begin{abstract}
Quatre publications viennent récemment d'analyser la translocation chromosomique $\mathrm{t}(2 ; 5)$ et le point de cassure $5 \mathrm{q} 35$ et de souligner leur valeur dans l'étude et le démembrement des lymphomes anaplasiques à grandes cellules $\mathrm{CD}^{+} 0^{+}$(ou $\mathrm{Ki}-1+$ ) [1, 2].

Étudiant quelques lignées tumorales porteuses de cette anomalie, Morris et al. [1] ont, en effet, mis en évidence un réarrangement du gène $N P M$ (nucléophosmine), situé en 5 q35, et la fusion de ce gène modifié avec un autre gène, $A L K$, situé en p23 sur le bras court du chromosome 2 (figure 1). Le gène $N P M$, identifié depuis 1989 [3], code pour une protéine impliquée dans le transport allerretour (shuttle) d'informations $\mathrm{du}$ nucléole au cytoplasme et dans l'assemblage terminal des protéines ribosomiques. Le gène $A L K$ (anaplastic lymphoma kinase) code pour une protéine membranaire présentant de grandes analogies avec des récepteurs de type tyrosine kinase.
\end{abstract}

Outre l'intérêt de fournir un nouvel exemple aux modèles déjà connus de cancérogenèse et de leucémogenèse fondés sur la fusion des gènes impliqués dans l'activation et la différenciation cellulaires (CML, PML, Burkitt...), l'analyse moléculaire de la translocation $\mathrm{t}(2 ; 5)$ concrétise sa valeur nosologique et fournit des outils pour l'identifier plus commodément. Elle permet aussi de mieux définir sa place dans les lymphomes anaplasiques à grandes cellules et de préciser ses rapports avec la maladie de Hodgkin et l'histiocytose
Étudiant 16 observations de lymphomes anaplasiques à grandes cellules (12 de phénotype T, 2 de type B et 1 de type nul), F. Bullrich et al. [2] signalaient un réarrangement du gène NPM dans un seul lymphome anaplasique à grandes cellules de type $\mathrm{T}$ et un seul lymphome de type B avec, dans ce dernier, une expression moléculaire un peu différente. La présence aussi rare de ce réarrangement constitue une surprise car, sur la foi d'études chromosomiques antérieures, il était implicitement admis que la translocation $t(2 ; 5)$ était présente dans près du quart des lymphomes anaplasiques à grandes cellules [4]. Un biais de recrutement peut sans doute rendre compte de cette différence.

En effet, la translocation $t(2 ; 5)$ a été principalement signalée dans les lymphomes anaplasiques à grandes cellules de l'enfant, comme sont d'origine infantile les lignées cellulaires (SU-DHL-1, SUP-M2, DEL, Karpas ) qui ont fourni la matière de ces études moléculaires. Quelle que soit la fréquence réelle du réarrangement, ces données chromosomiques et moléculaires confirment une notion pressentie depuis longtemps: l'hétérogénéité foncière des lymphomes anaplasiques à grandes cellules qui ne devaient leur individualisation qu'à leur expression histologique et surtout leur positivité pour les marqueurs de différenciation CD30, CD25 et EMA.

Également surprenante est la découverte d'un réarrangement du gène NPM dans deux des neuf cas de maladie de Hodgkin qu'ont étudiés
F. Bullrich et al. [2] et dans onze des cas analysés par K. Orscheschek et al. [5]. Cependant, ces résultats sont contredits par une étude récente de M. Ladanyi et al. qui ne trouvent pas de gène de fusion $N P M / A L K$ dans 40 cas de maladie de Hodgkin de différentes variétés [6]. Ces constatations, si elles sont confirmées, peuvent recouper la positivité pour le CD30 des cellules de Sternberg et fournir des aliments inattendus aux éternelles discussions sur l'histogenèse, l'homogénéité ou l'hétérogénéité de la maladie de Hodgkin.

Surtout, le réarrangement du gène NPM vient à point conforter la valeur signalétique du point de cassure 5 q35 que quelques auteurs regardent comme caractéristique de l'histiocytose maligne [7-9].

Il est, en effet, juste de signaler que si le point de cassure $5 \mathrm{q} 35$ marque la translocation $t(2 ; 5)$, la plus fréquente, il est également présent dans les translocations $t(3 ; 5)$ et $\mathrm{t}(5 ; 6)$, découvertes dans des affections d'expression clinique identique, ayant les traits d'une maladie systémique et comportant de nombreuses localisations extraganglionnaires. Ainsi, à l'appui de cette ressemblance, la lignée DEL porteuse de translocation $\mathrm{t}(5 ; 6)$ montre le même réarrangement du gène $N P M$ que les lignées $\mathrm{t}(2: 5)$ et exprime le même transcrit, bien que jusqu'à présent aucune anomalie n'ait été décelée sur le bras court du chromosome 2 [2].

Dans l'analyse de ces translocations, F. Bullrich et al. [2] indiquent que l'étude du gène NPM (taille molécu- 


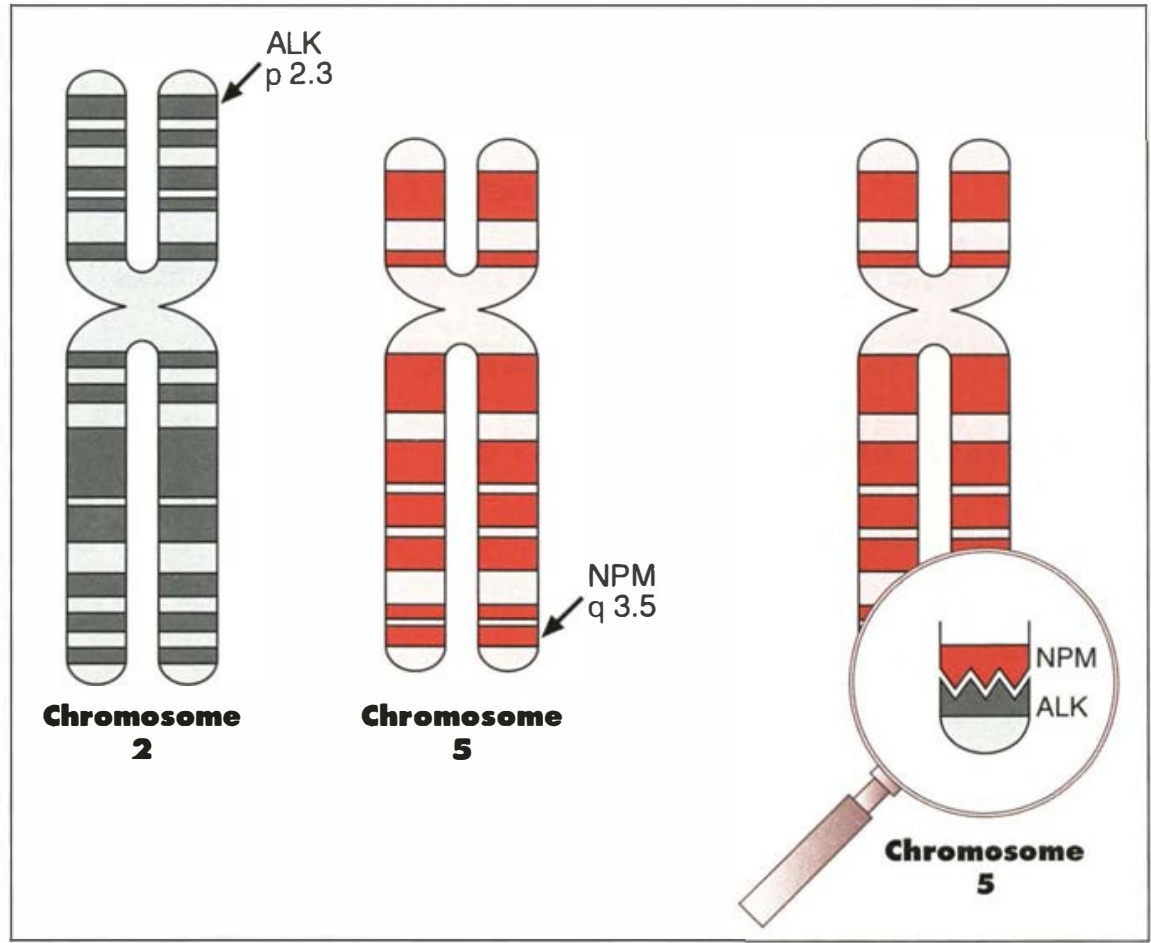

laire de 1,18 kb) fournit des données plus stables et précises que celles, plus aléatoires, du gène $A L K$ dont la taille et les transcrits ne sont pas encore connus avec précision.

Alors qu'il est fortement exprimé dans le placenta, le foie fotal, l'intestin grêle, le gène $A L K$, curieusement, n'est spontanément exprimé, ni dans les cellules hématopoiétiques, ni dans les cellules et les lignées lymphoïdes [1]. Ce comportement inattendu est susceptible de relancer une fois de plus la controverse sur l'origine lymphoïde, histiocytaire ou autre des lymphomes anaplasiques à grandes cellules $\mathrm{CD}^{2}{ }^{+}$. Cette querelle histogénétique paraît aujourd'hui dépassée depuis qu'il est établi que le réarrangement d'un seul gène comme celui du MLL (mixed lineage leukemia) associé à un unique point de cassure en 1 lq23 peut s'observer à la fois dans les leucémies lymphoblastiques et myéloblastiques [10].
Quoi qu'il en soit, il apparaît désormais clair que les anomalies moléculaires du gène $N P M$, en soulignant la valeur signalétique du point de cassure 5q35, marquent une étape importante dans le démembrement et une meilleure compréhension des lymphomes anaplasiques à grandes cellules $\mathrm{CD} 30^{+}$.

1. Morris SW, Kirstein MN, Valentine MB Dittmer KG, Shapiro DN, Saltman DL, Look AT. Fusion of a kinase gene, $A L K, t()$ a nucleolar protein gene, NPM, in non-Hodgkin's lymphoma. Science $1994 ; 263$ : 1281-4

2. Bullrich F, Morris SW, Hummel M, Pileri S, Stein H, Croce CM. Nucleophosmin (NPM) gene rearrangements in $\mathrm{Ki}-1$ positive lymphomas. Cancer Res 1994; 54: 2873-7.
Figure 1. Translocation $t(2 ; 5)$ observée dans quelques lymphomes anaplasiques à grandes cellules $\mathrm{CD} 3 \mathrm{O}^{+}$. Le gène ALK (anaplastic lymphoma kinase) situé sur le bras court du chromosome 2 (2p2.3) est transloqué sur le bras long du chromosome 5 (5q35) où est situé le gène NPM, à proximité du point de cassure $5 q 35$, identifié depuis plusieurs années. Dans la protéine hybride, la partie aminoterminale de la nucléophosmine serait liée au domaine catalytique d'ALK. La perte du contrôle de l'expression de cette kinase, qui ressemble aux récepteurs de type tyrosine kinase, contribuerait à la transformation maligne dans ces lymphomes.
3. Borer RA, Lehner CF, Eppenberger HM, Nigg EA. Major nucleolar proteins shuttle between nucleus and cytoplasm. Cell 1989; 56: 379 90.

4. Dekmezian R, Goodacre A, Cabanillas F. The 2;5 translocation: is it specific for anaplastic ( $\mathrm{Ki}$ 1) large cell lymphomas? Mod Pathol 1990; $3: 25$ (A).

5. Orscheschek K, Merz. H, Hell J, Binder T, Bartels H, Feller AC. Large-cell anaplastic lymphoma-specific translocation $(t 2 ; 5)(p 23 ; q 35)$ in Hodgkin's disease: indication of a common pathogenesis? Lancel $1995 ; 345: 87-90$.

6. Ladanyi M, Cavalchire G, Morris SW, Downing J Filipa DA. Reverse transcriptase polymerase chain reaction for $\mathrm{Ki}-\mathrm{l}$ anaplastic large cell lymphomaassociated $\mathrm{t}(2 ; 5)$ translocation in Hodgkin's disease. Am J Pathol 1994; 145 : 1296-300.

7. Nezelof C, Barbey S, Gogusev J, TerrierLacombe MJ. Malignant histiocvtosis: a distinctive CD30 positive clinicopathological entity associated with a chromosomal translocation involving 5q35. Sem Diagn Pathol 1992; 9: 75-89.

8. Nezelof $C$. The $5 q .35$ bp chromosomal abnormality characterizes certain CD30 positive anaplastic large cell lymphomas offering a new definition of malignant histiocytosis in childhood. Nouv Rev Fr Hematol 1993; 35: 46.3-7.

9. Cline MJ. Histiocytes and histiocytosis. Blood $1994 ; 84: 2840-53$

10. Clean ML. A promiscous oncogene in acute leukemia. N Engl J Med 1993; 329 : 958-9. 